\title{
Percutaneous transluminal septal myocardial ablation for hypertrophic obstructive cardiomyopathy: long term follow up of the first series of 25 patients
}

\author{
L Faber, A Meissner, P Ziemssen, H Seggewiss
}

\begin{abstract}
Objective-To determine the long term outcome in patients treated with percutaneous transluminal septal myocardial ablation (PTSMA) for hypertrophic obstructive cardiomyopathy (HOCM).

Design and setting-Observational, single centre study.

Patients-25 patients (13 women, 12 men, mean (SD) age 54.7 (15.0) years) with drug treatment resistant New York Heart Association (NYHA) class 2.8 (0.6) symptoms attributed to a high left ventricular outflow gradient (LVOTG) and a coronary artery anatomy suitable for intervention.

Intervention-PTSMA by injection of 4.1 (2.6) $\mathrm{ml}$ of alcohol (96\%) into 1.4 (0.6) septal perforator arteries to ablate the hypertrophied interventricular septum.

Outcome measures-During in-hospital follow up, enzyme rise, the frequency of atrioventricular conduction lesions requiring permanent DDD pacing, and in-hospital mortality were assessed. Long term follow up (30 (4) months, range 24-36 months) included symptoms, echocardiographic measurements of left atrial and left ventricular dimensions and function, and LVOTG.

Results-Mean postinterventional creatine kinase rise was 780 (436) U/1. During PTSMA 13 patents developed total heart block, permanent pacing being necessary in five of them. One 86 year old patient died from ventricular fibrillation associated with intensive treatment ( $\beta$ mimetic and theophylline) for coexistent severe obstructive airway disease. After three months, three patients underwent re-PTSMA because of a dissatisfactory primary result, leading to LVOTG elimination in all of them. During long term follow up, LVOTG showed sustained reduction (3 (6) $\mathrm{mm} \mathrm{Hg}$ at rest and 12 (19) $\mathrm{mm} \mathrm{Hg}$ with provocation) associated with stable symptomatic improvement (NYHA class $1.2(1.0)$ ) and without significant global left ventricular dilatation.

Conclusions-PTSMA is an effective non-surgical technique for reduction of symptoms and LVOTG in HOCM. Prospective, long term observations of larger populations are necessary in order to determine the definitive significance of the procedure.
\end{abstract}

(Heart 2000;83:326-331)

Keywords: hypertrophic obstructive cardiomyopathy; percutaneous transluminal septal myocardial ablation; left ventricular outflow tract gradient; myocardial contrast echocardiography

Department of

Cardiology, Heart

Center NRW,

Ruhr-University of

Bochum,

Georgstrasser 11,

D-32545 Bad

Oeynhausen, Germany

L Faber

A Meissner

P Ziemssen

H Seggewiss

Correspondence to: Prof Dr Seggewiss email: seggewiss.hubert@ t-online.de

Accepted 20 October 1999
Non-surgical catheter treatment of hypertrophic obstructive cardiomyopathy $(\mathrm{HOCM})^{1-8}$ was introduced in 1994. In patients symptomatic enough to be considered candidates for surgical myectomy ${ }^{10}$ alcohol induced percutaneous transluminal septal myocardial ablation (PTSMA) reduces symptoms and left ventricular outflow tract gradient (LVOTG). Longer term observations of PTSMA treated patients are still lacking because of the novelty of the procedure. Having previously reported our short term experience, ${ }^{5}$ we now report on the long term results in the first 25 patients treated with PTSMA in our institution.

\section{Patients and methods}

STUDY POPULATION

Between January 1996, when interventional treatment of HOCM was initiated in our institution (with the help of Professor U Sigwart, from the Royal Brompton Hospital, London, UK), and August 1996, 25 patients with a mean
(SD) disease duration of 7.4 (4.0) years underwent PTSMA. Detailed baseline data and inclusion criteria have been described elsewhere. ${ }^{25} 8$ In brief, we included patients with symptoms despite tolerable medical treatment who showed an LVOTG $>50 \mathrm{~mm} \mathrm{Hg}$ at rest or $>100 \mathrm{~mm} \mathrm{Hg}$ at provocation, and who had at least one septal branch suitable for intervention. Patients with coexistent cardiac abnormalities requiring surgery were excluded. Prior myectomy had been performed in three patients $(12 \%)$, and two patients (8\%) had not responded to previous DDD pacemaker implantation. Further baseline data are shown in table 1.

Each patient gave written informed consent before intervention after intensive discussion of the various treatment options, with special respect to the novelty of PTSMA and the absence of long term experience.

PRE-INTERVENTIONAL AND FOLLOW UP STUDIES The study protocol with pre- and postinterventional workup is shown in table 2. Echocardiographic measurements were obtained following 


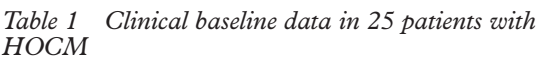

\begin{tabular}{lc}
\hline & $n(\%)$ \\
\hline Women & $13(52)$ \\
Family history of HOCM or sudden cardiac death & $10(40)$ \\
Complications of HOCM & $11(44)$ \\
$\quad$ History of syncopal attacks & $6(24)$ \\
History of acute left heart failure/pulmonary oedema & $10(40)$ \\
$\quad$ Documented supraventricular tachyarrhythmia & \\
Comorbidity & $3(12)$ \\
$\quad$ Pulmonary (COPD, restrictive abnormalities) & $5(20)$ \\
$\quad$ Systemic arterial hypertension & $7(28)$ \\
Treatment before PTSMA & $17(68)$ \\
$\quad$ B Blocker & $2(8)$ \\
$\quad$ Perapamil & $3(12)$ \\
$\quad$ Prior DDD pacemaker implantation & \\
\hline COPD, chronic obstructive pulmonary disease. &
\end{tabular}

the American Society of Echocardiography guidelines. ${ }^{11}$ LVOTGs were assessed by continuous wave Doppler echocardiography at rest and with a Valsalva manoeuvre. Mitral regurgitation and systolic anterior movement (SAM) of the mitral valve apparatus were graded semiquantitatively - that is, from $0=$ absent to $3=$ severe/with complete septal apposition. ${ }^{12}$ Symptom limited bicycle exercise tests in upright position were performed in patients with New York Heart Association (NYHA) functional class <IV and resting LVOTG $<100 \mathrm{~mm} \mathrm{Hg}$, starting with $25 \mathrm{~W}$ and increasing by $25 \mathrm{~W}$ every two minutes.

\section{INTERVENTION}

These first 25 patients underwent PTSMA as previously described by Sigwart and our own group ${ }^{125}$ with measurement of the left ventricular inflow tract pressure by a Brockenbrough catheter introduced transseptally. Aortic pressure was monitored by the percutaneous transluminal coronary angiography (PTCA) guiding catheter after exclusion of an aortic valve gradient. The LVOTG was assessed at rest and after a premature ventricular beat. All patients received a $4 \mathrm{~F}$ transfemoral pacemaker lead introduced into the right ventricular apical region, and $10000 \mathrm{IU}$ heparin intravenously as antithrombotic prophylaxis.

The presumed target vessel was then selectively intubated with a 0.014 inch guidewire via a $7 \mathrm{~F}$ or $8 \mathrm{~F}$ PTCA guiding catheter (fig 1). A short, slightly oversized over the wire balloon $(2.0-2.5 \mathrm{~mm})$ was introduced and inflated, and the distal vessel bed contrasted. After exclusion of dye reflux into the left anterior descending coronary artery, and provided probatory vessel closure by the inflated balloon had resulted in significant LVOTG reduction,

Table 2 Study protocol

\begin{tabular}{|c|c|c|c|}
\hline Before PTSMA & After PTSMA & 3 months after PTSMA & $\begin{array}{l}12 \text { months after PTSMA } \\
\text { and yearly thereafter }\end{array}$ \\
\hline $\begin{array}{l}\text { History } \\
\text { Clinical status } \\
\text { ECG } \\
\text { Lung function test } \\
\text { Bicycle stress test } \\
\text { Pulmonary artery catheter } \\
\text { Echocardiography } \\
\text { Diagnostic cardiac } \\
\text { catheterisation }\end{array}$ & $\begin{array}{l}\text { History } \\
\text { Clinical status } \\
\text { ECG } \\
\text { Enzyme monitoring }\end{array}$ & $\begin{array}{l}\text { History } \\
\text { Clinical status } \\
\text { ECG } \\
\text { Bicycle stress test } \\
\text { Pulmonary artery catheter } \\
\text { Echocardiography } \\
\text { (Diagnostic cardiac } \\
\text { catheterisation) }\end{array}$ & $\begin{array}{l}\text { History } \\
\text { Clinical status } \\
\text { ECG } \\
\text { Bicycle stress test } \\
\text { Echocardiography }\end{array}$ \\
\hline
\end{tabular}
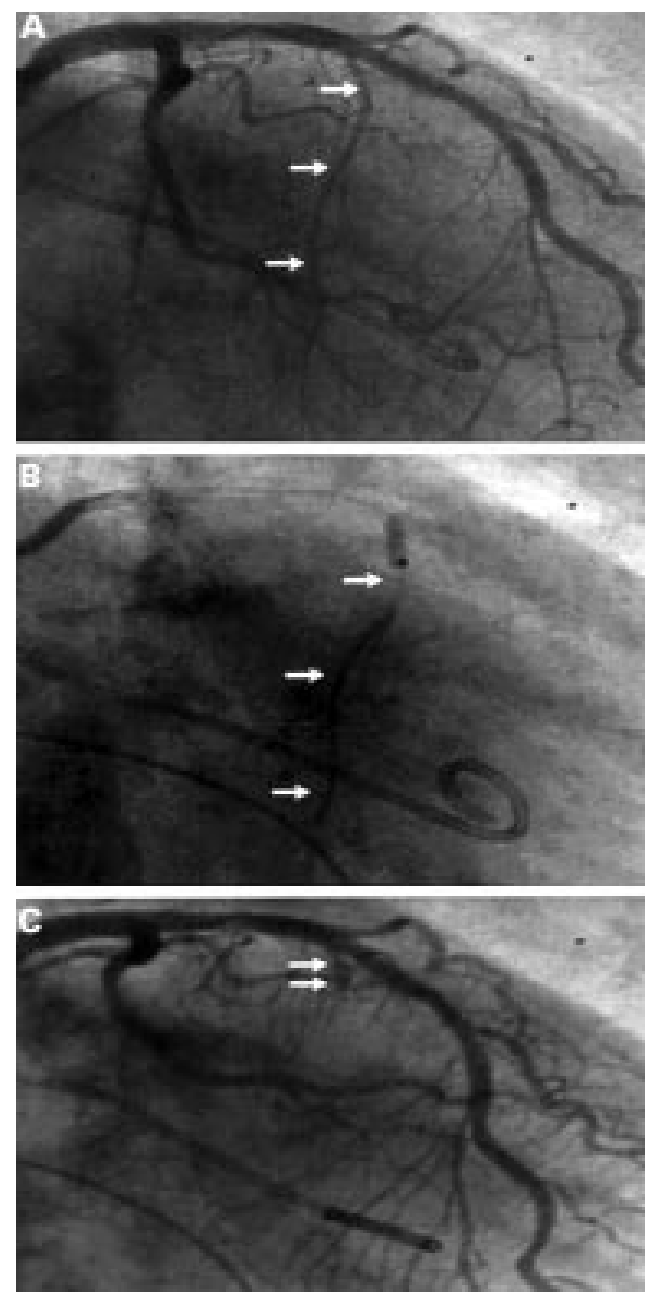

Figure 1 Coronary angiograms. (A) Identification of the target vessel in right anterior oblique position (arrows). (B) Injection of contrast dye to define the perfusion area and to exclude reflux into the left anterior descending coronary artery. (C) Final visualisation of the vessel stump after completed PTSMA

the alcohol (96\%) was injected. The balloon remained inflated for up to 10 minutes after the injection of alcohol in order to enhance tissue contact and to exclude alcohol reflux into the left anterior descending coronary artery. Finally, haemodynamic measurements were repeated (fig 2).

\section{FOLLOW UP STUDIES}

All patients were monitored on the coronary care unit for at least 48 hours. The vascular sheaths were removed after normalisation of the coagulation measurements. Cardiac enzymes and ECG controls were done every 4 hours. Before discharge, non-invasive follow up was performed as shown in table 2 . If possible, medical treatment was continued with a cardioselective $\beta$ blocker or, in case of contraindications, with a reduced dose of verapamil. After three and 12 months, and yearly thereafter, all patients underwent clinical and non-invasive follow up.

\section{STATISTICS}

Patient data were collected in a relational database (Filemaker 3.0, Claris) and analysed with the Statview 4.5 (Abacus Concepts) and 


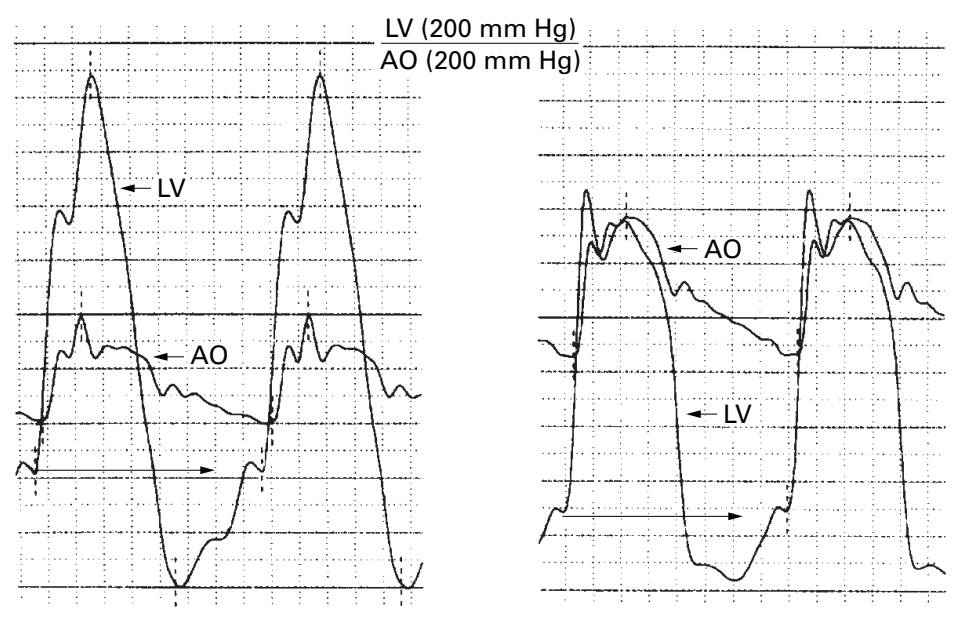

Figure 2 Haemodynamic treatment result of PTSMA. Pressure curves of left ventricular inflow tract $(L V)$ and aorta $(A O)$ before (left) and after (right) intervention with complete elimination of the LVOTG and reduction of left ventricular end diastolic pressure (lower arrows).

Winstat 3.1 (Kalmia) statistical software packages. Results of continuous variables are displayed as mean (SD). Student's $t$ test for paired samples were used for group comparisons (baseline and follow up measurements). Frequency distributions were assessed with the $\chi^{2}$ test. Analysis of variance was performed when comparing more than two groups. Differences were considered significant if the two tailed $\mathrm{p}$ value was $<0.05$.

\section{Results}

TECHNICAL ASPECTS, COMPLICATIONS, AND

ACUTE HAEMODYNAMIC RESULTS OF PTSMA

Since detailed information on the in-hospital course in these patients has already been published, ${ }^{5}$ only the main aspects are summarised here. Mean procedural time was 102 (33) minutes (range 46-173 minutes) and fluoroscopy time was 18 (13) minutes (range 6-45 minutes). The amount of alcohol injected was 4.1 (2.6) $\mathrm{ml}$ (range $1.5-11 \mathrm{ml}$ ) with $1.4(0.6)$ target vessels occluded (range 1-3, two vessels in seven patients, three vessels in one patient). Chest pain induced by alcohol injection could be managed by central analgesics.

Complete heart block developed in 13 patients $(52 \%)$; five of these $(20 \%)$ required permanent DDD pacemaker implantation because of sustained or intermittent conduction problems. The creatine kinase peak was 780 (427) U/1 (range 305-1810 U/1) after 11 (6) hours (range 4-24 hours) with an MB fraction of 103 (63) U/1 (range 45-281 U/1). One 86 year old patient, with coexistent severe pulmonary obstructive disease, died following exacerbation and intensive treatment with $\beta$ agonists and theophylline ${ }^{58}$ because of intractable ventricular fibrillation on the ninth day after a successful intervention. This was the first death in our series.

SHORT TERM FOLLOW UP AFTER THREE MONTHS After three months, 21 of the returning 24 patients $(88 \%)$ reported significant improvement of symptoms and exercise tolerance, with a mean NYHA class reduction from $2.8(0.6)$ to $1.4(1.1)(\mathrm{p}<0.001)$ and reduction of LVOTG to $20(17) \mathrm{mm} \mathrm{Hg}$ at rest and 56 (48) $\mathrm{mm} \mathrm{Hg}$ with provocation (table 3). SAM and obstruction associated mitral regurgitation decreased in a parallel way. Maximum tolerated workload improved from 67 (74) W to $111(50) \mathrm{W}(\mathrm{p}<0.01)$.

In three patients, however, a re-PTSMA had to be performed at that time $^{5}$ because of LVOTG recurrence or persistence in association with disabling class III symptoms. This second intervention, in two patients already guided by intraprocedural contrast echocardiography, ${ }^{7}$ led to symptomatic and haemodynamic improvement in all cases. The short term follow up period after all these interventions was free from cardiac events.

Figure 3 shows the morphological results of treatment with PTSMA.

LONG TERM FOLLOW UP

All patients underwent non-invasive follow up after 12 months and at least one additional re-evaluation thereafter. After 12 months clinical success was sustained with a mean NYHA class of 1.4 (1.1) and a maximum workload of 113 (39) W with upright bicycle exercise $(\mathrm{p}<0.001 v$ baseline $)$. With the exception of one patient who developed sustained atrial fibrillation and was successfully cardioverted, the first year after PTSMA again was free from cardiac events. All patients reported satisfactory symptomatic improvement without any need for treatment intensification. Two patients were not taking any cardiac medication, 18 were treated with low dose $\beta$ blockers (metoprolol $50-100 \mathrm{mg} /$ day or comparable), and four were treated with verapamil (240$360 \mathrm{mg} / \mathrm{day})$.

The LVOTG showed further regression as compared to short term follow up, with a mean of 9 (13) $\mathrm{mm} \mathrm{Hg}$ at rest and 29 (43) $\mathrm{mm} \mathrm{Hg}$ with provocation (fig 4). All patients had a

Table 3 Clinical end echocardiographic evolution after PTSMA

\begin{tabular}{lllll}
\hline & & $\begin{array}{l}\text { 3 months after } \\
\text { PTSMA }\end{array}$ & $\begin{array}{l}\text { 12 months after } \\
\text { PTSMA }\end{array}$ & Last control \\
\hline NYHA functional class & $2.8(0.6)$ & $1.4(1.1)^{\star \star \star}$ & $1.4(1.1)^{\star \star \star}$ & $1.2(1.0)^{\star \star \star}$ \\
Maximum workload (W) & $67(74)$ & $111(50)^{\star \star}$ & $113(39)^{\star \star \star}$ & $105(40)^{\star \star}$ \\
Left atrial diameter (mm) & $48.0(8.1)$ & $45.7(8.1)^{\star}$ & $44.6(6.2)^{\star \star}$ & $46.3(8.5)^{\star}$ \\
Left ventricular end diastolic diameter (mm) & $46.6(5.3)$ & $48.8(6.1)$ & $50.0(6.9)^{\star}$ & $48.8(6.1)$ \\
Septal thickness (mm) & $21.9(3.5)$ & $17.0(6.5)^{\star \star \star}$ & $12.7(3.5)^{\star \star \star}$ & $11.7(2.7)^{\star \star \star}$ \\
Left ventricular posterior wall thickness (mm) & $14.3(2.6)$ & $12.3(5.5)$ & $11.3(2.0)^{\star \star}$ & $11.7(2.2)^{\star \star}$ \\
SAM (grade 1-3) & $2.4(0.8)$ & $0.7(0.9)^{\star \star \star}$ & $0.2(0.4)^{\star \star \star}$ & $0.3(0.4)^{\star \star \star}$ \\
Mitral regurgitation (grade 1-3) & $0.9(0.5)$ & $0.5(0.5)^{\star}$ & $0.4(0.5)^{\star}$ & $0.5(0.5)^{\star}$ \\
LVOTG at rest (mm Hg) & $60(38)$ & $20(17)^{\star \star \star \star}$ & $9(13)^{\star \star \star \star}$ & $3(6)^{\star \star \star \star}$ \\
LVOTG with provocation (mm Hg) & $147(56)$ & $56(48)^{\star \star \star \star}$ & $30(43)^{\star \star \star \star}$ & $12(19)^{\star \star \star \star}$ \\
\hline
\end{tabular}

Data presented as mean (SD); $\mathrm{p}<0.05 ;{ }^{\star \star} \mathrm{p}<0.01{ }^{\star \star \star} \mathrm{p}<0.001 ;{ }^{\star \star \star \star} \mathrm{p}<0.0001$ versus baseline values. 

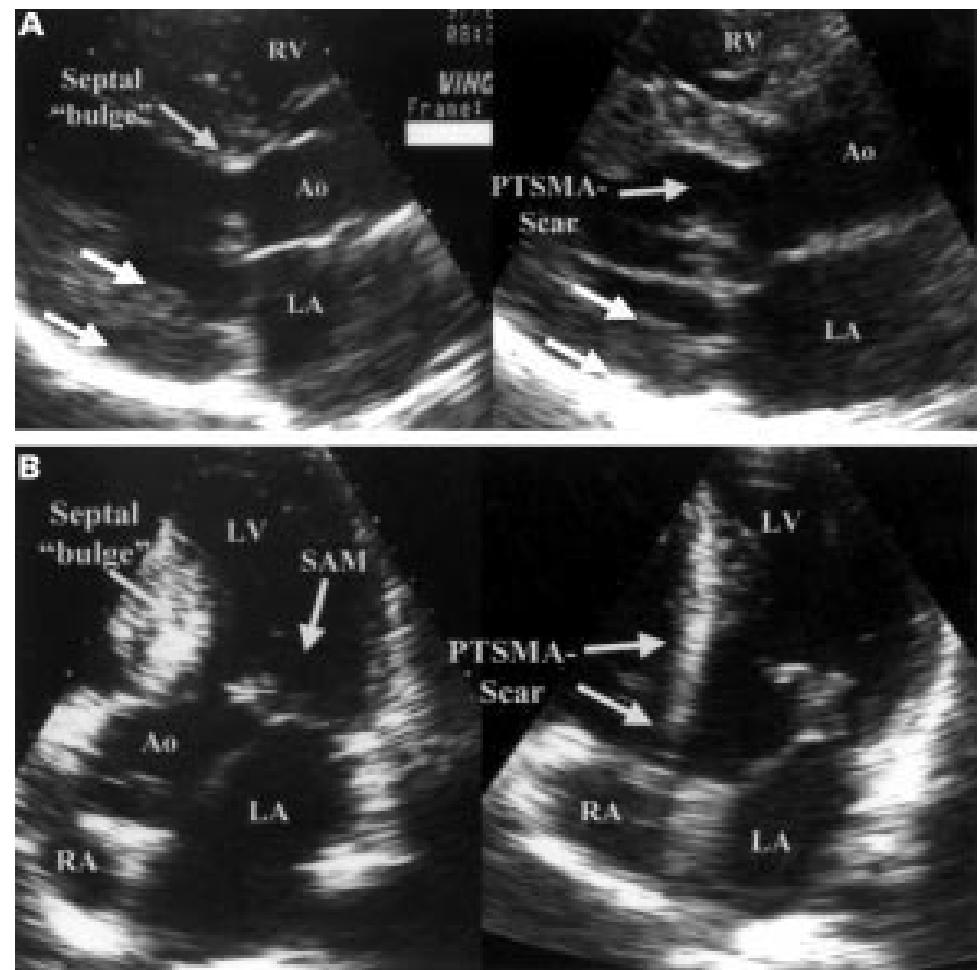

Figure 3 Morphological results of PTSMA. Parasternal long axis view $(A)$ and apical four chamber view (B) before (left) and after (right) PTSMA, showing the myectomy like subaortic scar leading to widening of the LVOT. Posterior wall of the left ventricle (LV) also shows regression of hypertrophy (lower arrows in $A$ ). Ao, aorta; $R V$, right ventricle; $L A$, left atrium. patients (59 and 70 years old) with coexistent systemic arterial hypertension and metabolic syndrome (body mass index 29.0 and $30.0 \mathrm{~kg} /$ $\mathrm{m}^{2}$, respectively); both successfully cardioverted. In another patient, also with metabolic syndrome (body mass index $30.0 \mathrm{~kg} / \mathrm{m}^{2}$ ), paroxysmal atrial fibrillation was noticed as before PTSMA. Two other patients remained in chronic atrial fibrillation. Left atrial diameter did not decrease, or gradually increased, in these five patients with atrial tachyarrhythmias. In the other 19 patients a stable sinus rhythm was present.

The five patients with a procedure related DDD pacemaker implantation did not differ from those with undisturbed atrioventricular conduction in terms of haemodynamic efficacy of the procedure (LVOTG elimination in all); however, a slightly higher NYHA class (1.3 $(1.0)$ v $1.0(1.0)$ ), presumably partly attributable to their advanced age (61 (13) $v 53$ (15) years; no statistical tests performed because of the small number) was reported. In two of them the pacemaker could be switched to a VVI backup stimulation mode because of stable recovery of the atrioventricular conduction at three and 12 months' follow up.

\section{Discussion}

Symptoms and disease manifestations in patients with HOCM include dyspnoea, angina, palpitations, syncope, heart failure, and sudden cardiac death, with obstruction to left ventricular outflow, diastolic dysfunction, and rhythm disturbances being the underlying causes. ${ }^{910}$ Symptomatic patients not only suffer from impaired quality of life but also seem to have an adverse prognosis. ${ }^{13}$ Medical treatment and surgical myotomy-myectomy have been the therapeutic options for decades since the late $1950 \mathrm{~s},{ }^{9}{ }^{10} 14^{16}$ the latter reserved for severely symptomatic patients (NYHA class III or IV), and both predominantly aiming at LVOTG reduction.

Atrioventricular sequential pacing and catheter treatment by interventional septal ablation have recently emerged as new non-medical treatment options. ${ }^{1-8}{ }^{17-19}$ In the limited number of reports published to date, DDD pacing reduced the LVOTG by $50 \%$. Further impairment of diastolic left ventricular filling caused by excessive shortening of the atrioventricular interval, ${ }^{19}$ and the impossibility to predict the LVOTG response to chronic artificial preexcitation by acute testing, are the main problems with this form of treatment. Furthermore, after cessation of pacing, symptoms and LVOTGs frequently recur, making unlimited pacing necessary. A remodelling process that leads to anatomic widening of the LVOT could not consistently be shown. ${ }^{20}$

The localised "therapeutic infarction" 21 induced by PTSMA as a result of ethanol injection into a septal perforator artery leads to thinning of the involved septal area, expands the left ventricular outflow tract, and eliminates or reduces systolic anterior movement of the mitral valve and outflow obstruction. ${ }^{1-8}$ After a local remodelling process, the morphologic as well as the haemodynamic result resembles

Figure 4 Evolution of the LVOTG after PTSMA. $\star_{p}<0.05 ;{ }^{\star *} p<0.01 ; \star \star * p<0.0001$ versus prior measurement. 
that of a surgical myectomy ${ }^{5}$ with a channel like scar in place of the septal bulge. A significant LVOTG reduction is associated with a rise in exercise capacity by one or two NYHA classes, improvement of left ventricular diastolic function, reduction of SAM associated mitral regurgitation, and reduction of left atrial diameter. ${ }^{1-3}$ 5-8

The first preliminary studies leading to this catheter based imitation of surgical myectomy date from the 1980s. ${ }^{122}$ In 1994, probatory balloon occlusion of septal branches of the left coronary artery, ${ }^{23}$ leading to a transient, ischaemia induced LVOTG reduction, was reported. In 1995, Sigwart ${ }^{1}$ published the first report on definitive alcohol induced septal reduction in three severely symptomatic patients. The first series, covering up to 91 patients observed over periods of less than 12 months, ${ }^{84}$ showed promising results concerning symptoms, LVOTG reduction, complication rates, and mid term clinical course.

The present study adds a longer term perspective to these reports. The main findings are, firstly, that reduction of septal thickness, and consecutively outflow gradient, continues over a 12 month period, presumably because of ongoing fibrosis and shrinking of the PTSMA induced scar. The highest net gain in outflow tract diameter caused by septal reduction occurred between three and 12 months' follow up. Our previous experience concerning ongoing LVOTG reduction until mid term follow up is thus confirmed,,$^{824}$ as well as our strategy of waiting for at least three months before considering a re-PTSMA or other non-medical treatment options.

Our second finding was that progressive left ventricular dilatation was not observed, thus the remodelling process seems to remain limited to the region of intervention. This finding is important in view of those patients with spontaneous LVOTG elimination and left ventricular dilatation, which carry a particularly bad prognosis. ${ }^{25}$ Surprisingly, not only did septal hypertrophy decrease as a consequence of the therapeutic infarction, but also left ventricular posterior wall thickness. ${ }^{8}$ This may be caused by relief of the pressure overload following LVOTG reduction, which in turn indicates that the hypertrophic process in HOCM may not be completely independent of left ventricular afterload.

Taking into account the fact that the complete learning phase is present in this series, PTSMA seems to be a quite safe procedure. Complications, however, have to be considered as previously reported-that is, thoracic discomfort, atrioventricular conduction disturbances, ventricular tachyarrhythmias, and death. ${ }^{58}$ To date, the in-hospital mortality rate of PTSMA has come down to $1.5 \%$ $(3 / 201) .^{24}$

During long term follow up, despite LVOTG elimination, symptomatic improvement, and lack of major adverse events, atrial tachyarrhythmia seems to be a persistent problem especially in patients with coexistent systemic arterial hypertension. Lifestyle counselling and medical treatment aimed at cardiovascular risk

\section{Acronyms and abbreviations}

HOCM: Hypertrophic obstructive cardiomyopathy

LVOT: Left ventricular outflow tract

LVOTG: Left ventricular outflow tract gradient

NYHA: New York Heart Association

PTSMA: Percutaneous transluminal septal myocardial ablation

SAM: Systolic anterior movement of the mitral valve

factor management thus seems to be appropriate in this patient group. On the other hand, a successful PTSMA at best changes HOCM into its non-obstructive variant. It is thus not surprising that diastolic dysfunction, increased atrial load, and a tendency to atrial arrhythmias persist to a certain extent and continue to pose therapeutic problems. ${ }^{910}$

Finally it should be mentioned that in these first 25 patients haemodynamic testing by probatory balloon occlusion with its low predictive value was the only available method for target vessel selection. In 11 patients more than one target vessel had to be occluded for satisfactory LVOTG reduction, either in one session or by re-PTSMA, and myocardial loss as assessed by the enzyme peaks was higher than actually observed $^{5824}$ with intraprocedural echocardiographic monitoring.

It may therefore be speculated that the beneficial effects of left ventricular unloading in some of these patients were partially offset by worsening of left ventricular diastolic properties following rather extensive septal scarification. With combined probatory balloon occlusion and echocardiographic monitoring, a more precise targeting of the myocardial necrosis is possible, resulting in smaller scars created by occlusion of the one "culprit vessel" ${ }^{\prime-8}$ responsible for LVOTG formation.

\section{LIMITATIONS}

Several limitations have to be considered in this report. M mode measurements of left atrial and left ventricular dimensions may not sample exactly maximum or minimum wall thickness, may be subject to angle errors, and were not performed blinded with respect to treatment status. Doppler measurements of the LVOT gradient are not very accurate in the lower range. Short, self limiting episodes of atrial or ventricular tachyarrhythmias may have been missed because only sustained or documented arrhythmia, or both, were included for analysis in this report. Finally, the patients were not randomly assigned to interventional treatment but by individual indication. A comparison with established forms of non-medical treatment of HOCM is thus not possible.

\section{CONCLUSIONS}

PTSMA is a promising interventional treatment modality for patients with HOCM who do not show satisfactory response to medical 
treatment. Elimination or a substantial reduction of LVOTG and associated abnormalities, without the operative trauma, is possible, similar to the effect of surgical myectomy. Global systolic function seems to be preserved, although the effect on prognosis remains to be assessed. A prospective registry of all interventionally treated HOCM patients will be very helpful in clarifying these issues.

1 Sigwart U. Non-surgical myocardial reduction for hypertrophic obstructive cardiomyopathy. Lancet 1995;346:211trop

2 Gleichmann U, Seggewiss H, Faber L, et al. Kathetertherapie der hypertrophen obstruktiven Kardiomyopathie. Dtsch Med Wochenschr 1996;121:679-85.

3 Knight CJ, Kurbaan AS, Seggewiss H, et al. Non-surgical septal reduction for hypertrophic obstructive cardiomyopathy: Outcome in the first series of patients. Circulation 1997;95:2075-81.

4 Kuhn H, Gietzen F, Leuner C, et al. Induction of subaortic septal ischemia to reduce obstruction in hypertrophic obstructive cardiomyopathy. Eur Heart $\mathcal{F}$ 1997;18:846-51.

5 Seggewiss H, Gleichmann U, Faber L, et al. Percutaneous transluminal septal myocardial ablation in hypertrophic obstructive cardiomyopathy: acute results and 3-months follow-up in 25 patients. $\mathcal{F}$ Am Coll Cardiol 1998;31:252-8.

6 Faber L, Seggewiss H, Fassbender D, et al. Catheter treatment in hypertrophic obstructive cardiomyopathy: identification of the perfusion area of septal branches by myocardial contrast echocardiography [abstract]. Eur Heart myocardial contrast ech

7 Faber L, Seggewiss H, Fassbender D, et al. Guiding of PTSMA in obstructive hypertrophic cardiomyopathy by myocardial contrast echocardiography: a case report. four nal of Interventional Cardiology 1998;11:443-8.

8 Faber L, Seggewiss H, Gleichmann U. Percutaneous transluminal septal myocardial ablation in hypertrophic obstructive cardiomyopathy: results with respect to intraprocedural myocardial contrast echocardiography. Circulation 1998;98:2415-21

9 Spirito P, Seidman CE, Mc Kenna WJ, et al. The management of hypertrophic cardiomyopathy. $N$ Engl $\mathcal{F}$ Med 1997;336:775-85.

10 Wigle DE, Rakowski H, Kimball BP, et al. Hypertrophic cardiomyopathy: clinical spectrum and treatment. Circulation 1995;92:1680-92.

11 Sahn DJ, De Maria AN, Kisslo J, et al. The committee on M-mode standardization of the American Society of EchoM-mode standardization of the American S
cardiography. Circulation 1978;58:1072-83.
12 Pollick C, Rakowsky H, Wigle ED. Muscular subaortic stenosis: the quantitative relationship between SAM and the pressure gradient. Circulation 1984;69:43-9.

13 Takayi E, Yamakado T, Nakano T. Prognosis of completely asymptomatic adult patients with HOCM . F Am Coll Cardiol 1999;33:206-11.

14 Morrow AG, Brockenbrough EC. Surgical treatment of idiopathic hypertrophic subaortic stenosis: Technique and hemodynamic results of subaortic ventriculotomy. Ann Surg 1961;154:181-9.

15 Robbins RC, Stinson EB, Daily PO. Long-term results of left ventricular myotomy and myectomy for obstructive hypertrophic cardiomyopathy. I Thorac Cardiovasc Surg 1996;111:86-94.

16 Mc Cully RB, Nishimura RA, Tajik AJ, et al. Extent of clinical improvement after surgical treatment of hypertrophic obstructive cardiomyopathy. Circulation 1996;94:467-71.

17 Fananapazir L, Epstein ND, Curiel RV, et al. Long-term results of dual-chamber (DDD) pacing in obstructive hypertrophic cardiomyopathy. Circulation 1994;90:273142 .

18 Kappenberger L, Linde C, Daubert C, et al and the PIC Study Group. Pacing in hypertrophic obstructive cardiomyopathy. Eur Heart f 1997;18:1249-56.

19 Nishimura RA, Hayes DL, Ilstrup D, et al. Effect of dual-chamber pacing on systolic and diastolic function in patients with hypertrophic cardiomyopathy: acute Doppler echocardiographic and catheterization hemodynamic study. F Am Coll Cardiol 1996;27:421-30.

20 Gadler F, Linde C, Ryden L. Rapid return of LVOT obstruction and symptoms following cessation of longterm AV synchronous pacing for HOCM. Am f Cardiol 1999;83:513-8.

21 Braunwald E. Induced septal infarction: a new strategy for hypertrophic obstructive cardiomyopathy. Circulation 1997; 95:1981-2.

22 Brugada P, De Swart H, Smeets JLRM, et al. Transcoronary chemical ablation of ventricular tachycardia. Circulation 1989;79:475-82.

23 Gietzen F, Leuner C, Gerenkamp T, et al. Relief of obstruction in hypertrophic cardiomyopathy by transient occlusion of the first septal branch of the left coronary artery [abstract]. Eur Heart $\mathcal{F}$ 1994;15:125.

24 Seggewiss H, Faber L, Gleichmann U, et al. Remodeling after percutaneous transluminal septal myocardial ablation in HOCM results in ongoing outflow tract gradient reduction [abstract]. F Am Coll Cardiol 1999;33(suppl 2):A490.

25 Hina K, Kusachi S, Iwasaka K, et al. Progression of left ventricular enlargement in patients with hypertrophic cardiomyopathy: incidence and prognostic value. Clin Cardiol 1993;16:403-7. 